

\title{
Lecture croisée dans le rapport de la Cour des comptes de 2019 sur l'évaluation des services publics en ligne au Maroc
}

\author{
Karim Zaouaq
}

\section{- To cite this version:}

Karim Zaouaq. Lecture croisée dans le rapport de la Cour des comptes de 2019 sur l'évaluation des services publics en ligne au Maroc. JiL Journal of Human \& Social Sciences, 2020, No. 64, pp. 159-170. hal-02860300

\section{HAL Id: hal-02860300 https://hal.science/hal-02860300}

Submitted on 8 Jun 2020

HAL is a multi-disciplinary open access archive for the deposit and dissemination of scientific research documents, whether they are published or not. The documents may come from teaching and research institutions in France or abroad, or from public or private research centers.
L'archive ouverte pluridisciplinaire HAL, est destinée au dépôt et à la diffusion de documents scientifiques de niveau recherche, publiés ou non, émanant des établissements d'enseignement et de recherche français ou étrangers, des laboratoires publics ou privés. 
Lecture croisée dans le rapport de la Cour des comptes de 2019 sur l'évaluation des services publics en ligne au Maroc'1.

\section{قراءة متقاطعة في تقرير المجلس الأعلى للحسابات لسنة 2019 حول تقييبم الخدمات على}

Dr. Karim Zaouaq/Université Hassan II de Casablanca-Maroc

د.كريم زواق/ جامعة الحسن الثاني بالدار البيضياء، المغرب

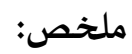

من المؤكد أن استخدام الإدارات المغربية لتكنولوجيا المعلومات والاتصالات قد تطور من خلال السياسات المعتمدة في هذا المجال. ومع ذلك، فإن التعبئة المؤسساتية لصالح هذه المتطلبات الرقمية لم يرق إلى مستوى توقعات مستخدمي الخدمات

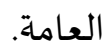

لهذا، عمل المجلس الأعلى للحسابات على جرد التطورات المسبلة في الخدمات العامة عبر الإنترنت، والذي انبثق عنه تقرير نشر في مايو 2019 مكن من تشخيص الخدمات العامة الرئيسية عبر الإنترنت في المغرب وإخضاعها لتحليل معمق فيما يتعلق بحكامتها.

ورغم ذلك، فإن البيانات الواردة في هذا التقرير لا تزال غير مكتملة بسبب المنهج الذي تم اعتماده وكذلك القياس المحدود لرضا الأطراف المعنية بتوفير الخدمات العامة عبر الإنترنت. الكلمات المفتاحية:الخدمات العامة عبر الإنترنت؛ الحكومة الإلكترونية؛ البيانات العامة؛ تفاعل؛ إعلام. 


\section{RÉSUMÉ}

Le recours des administrations marocaines aux technologies de l'information et de la communication a certes évolué à travers les multiples politiques conçues dans ce domaine. Mais, le déploiement institutionnel en faveur de telles exigences numériques a été en deçà des attentes des usagers des services publics.

Partant, la Cour des comptes a œuvré à dresser un état des lieux de l'évolution des services publics en ligne, ce qui a débouché sur la publication au cours du mois de mai 2019d'un rapport qui a établi un diagnostic unique des principaux services publics en ligne offerts au Maroc et les a soumis à une analyse approfondie en termes de gouvernance.

Toutefois, les données de ce rapport demeurent incomplètes en raison entre autres de la démarche méthodologique adoptée ainsi que de la prise en compte limitée du taux de satisfaction des parties concernées par les prestations des services publics en ligne.

MOTS CLÉS :Services publics en ligne- e-gouvernement- données publiques- interaction- information.

\section{Introduction}

«Le chemin vers la transparence, l'inclusion et la responsabilité n'est pas simple à emprunter, dans la mesure où il implique un changement de paradigme qui place les citoyens au cœur des politiques publiques et du fonctionnement concret des administrations publiques » Angel Gurría, Secrétaire Général de l'OCDE ${ }^{1}$

L'accessibilité aux services publics en ligne est une condition sine qua non cruciale pour une garantie effective de certains principes et dispositions énoncés par la Constitution de 2011, à savoir le principe de l'égal accès des citoyennes et citoyens à ces services (art. 154), le devoir incombant aux services publics d'« être à l'écoute de leurs usagers et d'assurer le suivi de leurs observations, propositions et doléances » (art. 156), ainsi que le droit des citoyennes et des citoyens d' « accéder à l'information détenue par l'administration publique, les institutions élues et les organismes investis d'une mission de service public » (art. 27).

${ }^{1}$ L'Organisation de coopération et de développement économique (OCDE), L'OCDE : un partenaire pour un gouvernement ouvert, p. 9. Disponible en ligne sur <http://www.oecd.org/mena/governance/Open-Government-brochure-fr.pdf >. 


\section{0 العدد 64 ـ هايرو}

La multiplication des plateformes et sites web publics, le développement des services qui y sont offerts et l'amélioration de l'accessibilité des usagers à ces derniers, participent généralement d'une optimisation du service public. De fait, il est reconnu que les services en ligne contribuent à titre d'exemple à « la réduction de moitié du délai de remboursement d'un trop-perçu par l'administration fiscale ${ }^{1}$ », de même que « la disparition du guichet au profit du traitement des requêtes en ligne réduit la possibilité de discrimination entre les usagers $^{2}$ ». Dans ce sens, «Andersen $\left(2009^{3}\right)$, en mesurant l'impact d'un indicateur d'e-administration sur un indicateur de corruption pour 140 pays entre 1996 et 2006, a montré que lorsqu'un pays passe du dernier au premier décile en termes d'e-administration, il gagne en moyenne deux déciles en termes d'absence de corruption ${ }^{4}$, tout en mettant en évidence « une corrélation positive et statistiquement significative de 0,46 entre les usages de l'administration en ligne et les indicateurs de transparence ${ }^{5} »$.

De ce fait et à la lumière des évolutions institutionnelles qu'a connues le Maroc dans le domaine de l'egouvernement, depuis l'adoption en 2009 de la stratégie Maroc Numérique 2013 jusqu'à l'institution de I'Agence de développement du digital, la Cour des comptes a jugé pertinent d'établir un rapport d'évaluation des services publics en ligne au Maroc. Ce dernier qui a été publié en mai 2019 dresse un état des lieux des progrès et contraintes pesant sur un développement effectif des services en ligne au Maroc, tout en présentant certaines recommandations visant à en améliorer l'accessibilité et la gouvernance. Mais, la démarche méthodologique adoptée et les données recueillies par la Cour laisse planer quelques insuffisances et limites.

\section{I- Méthodologie}

Pour la confection de son rapport, la Cour des comptes a fait usage de plusieurs approches méthodologiques en matière de collecte et d'analyse des données (1), tout en faisant appel à une multitude de parties prenantes publiques directement concernées par la question des services publics en ligne (2).

\footnotetext{
1Algan Yann, Maya Bacache-Beauvalletet Anne Perrot. «Administration numérique », Notes du conseil d'analyse économique, vol. 34, no. 7, 2016, p. 5 .

Ilbid.

${ }^{3}$ T. Andersen, «E-Government as an Anti-Corruption Strategy », Information Economics and Policy, n²1, 2009, pp. 201-210. Cité in : Yann Algan, Maya Bacache-Beauvallet, et Anne Perrot, ibid., p. 5

${ }^{4}$ Yann Algan, Maya Bacache-Beauvallet, et Anne Perrot, «Administration numérique », op. cit., pp. 5-6.

5lbid., p. 6.
} 


\section{0}

\section{هركز جيل البصث المانمي}

\section{1- Les données}

Certes, la Cour des comptes s'est basée, dans sa méthodologie d'évaluation des services publics en ligne, sur plusieurs indexes internationaux, reconnus par la plupart des États et des parties prenantes, dont l'EGovernment Development Index (EGDI), établi par l'Organisation des Nations Unies depuis 2003, mais il a négligé d'autres indicateurs importants, tels l'indicateur relatif à l'économie et à la société numérique (Digital Economy and Society Index, DESI) déployé par la Commission européenne depuis 2015.

En fait, contrairement à l'indice de développement de l'e-gouvernement (E-Government Development Index, EGDI) qui «s'appuie sur une moyenne pondérée des scores normalisés obtenus sur les trois dimensions suivantes: offre d'administration en ligne (Online Service Index, OSI), infrastructures numériques et capital humain ${ }^{1}$ », l'index DESI « ne résume pas l'état des lieux à l'offre des services dématérialisés ${ }^{2}$ », mais il dépasse ce cadre en tenant compte notamment des « usages de l'administration en ligne, tant par les particuliers que par les entreprises ${ }^{3}$ ». En effet, « le profil numérique des États étudiés dans ce dernier index est établi à partir de trente indicateurs couvrant cinq domaines : la connectivité (couverture, rapidité et coût du haut débit), les compétences numériques, les activités réalisées en ligne (banque, achats, lecture de la presse, etc.), l'intégration des technologies numériques par les entreprises et les services publics dématérialisés (administration et santé en ligne $)^{4} »$.

Pour sonder le degré de communication interactive entre les portails publics en ligne et les usagers, la Cour des comptes s'est contentée d'une observation des services offerts au niveau de ces portails, ce qui lui a permis de relever que très peu qui proposent une fonctionnalité de sondage d'opinion ou un jeu de questions/réponses instantanées entre les usagers et l'administration (un seul portail chacun), voire qu'aucun portail ne propose le renseignement d'un questionnaire de satisfaction par rapport à la qualité du site et des services rendus.

Dans ce sillage, la Cour s'est appuyée sur l'enquête effectuée en 2017 par l'Agence nationale de réglementation des télécommunications sur l'accès et l'usage des technologies de l'information et de la communication par les

'Ibid., p. 3.

${ }^{2}$ Ibid.

3lbid.

${ }^{4}$ lbid. 
individus et les ménages ${ }^{1}$. Cependant, la Cour des comptes n'a fait ressortir que peu de données de cette enquête, notamment celles relatives au fait que « le taux de pénétration d'internet chez les ménages marocains est passé de 20\% en 2009 à 70\% en 2017 et que l'utilisation d'internet pour interagir avec l'administration est toutefois limitée en ce sens qu'en 2017 aussi seulement 11,1\% des internautes au Maroc ont utilisé internet pour interagir avec les administrations publiques ${ }^{2}$ ». Mais, l'enquête en question qui a été réalisée sur le terrain du 12 février au 12 mars 2018 et qui a ciblé un échantillon de 12.000 ménages et individus résidant en milieux urbain et rural dans les 12 régions du Royaume et les individus âgés de 5 ans et plus ${ }^{3}$, recèle d'autres données non moins importantes qui n'ont pas été rapportées par la Cour des comptes, notamment les usages faits de l'internet par les individus, lesquels englobent à une proportion d'environ $12,4 \%$ des internautes interrogés l'obtention des informations auprès d'administrations publiques et à un taux de 11,5\% l'interaction avec les administrations publiques ${ }^{4}$. Cette enquête évoque également les contenus souhaités par les internautes marocains sur les sites web nationaux consultés et souligne que $29,3 \%$ de ces internautes cherche le contenu sur le gouvernement et l'administration (E-gov/emploi) ${ }^{5}$.

\section{2- Les parties sollicitées}

En ce qui concerne les parties sollicitées pour avis par la Cour des comptes dans le cadre de ce rapport, il y a lieu de souligner que l'approche de cette institution fût restrictive dans la mesure où seule une sélection de ministères et d'administrations publiques ont été invités à exprimer leur vision sur la question des services publics en ligne, en fournissant des réponses aux questions qui leur ont été adressées dans un questionnaire. Là étant, l'identité des parties interrogées n'a pas été divulguée, en ce sens que le rapport ne renferme que les noms des administrations qui ont fourni une réponse au questionnaire, à savoir le Ministère de l'industrie, de l'investissement, du commerce et de l'économie numérique, l'Agence nationale de réglementation des télécommunications et le Ministère de la réforme de l'administration et de la fonction publique, alors que les

${ }^{1}$ Voir<https://www.anrt.ma/indicateurs/etudes-et-enquetes/enquete-annuelle-marche-des-tic>.

${ }^{2}$ Cour des Comptes, Évaluation des services publics en ligne, mai 2019.

${ }^{3}$ Agence nationale de réglementation des télécommunications, Usages des TIC dans les ménages et par les individus, Année 2017: Synthèse des résultats, septembre 2018, p. 6. Disponible en ligne sur $<$ https://www.anrt.ma/sites/default/files/publications/enquete-tic-2017.pdf >.

${ }^{4}$ Ibid., p. 52.

IIbid., p. 53. 
acteurs publics qui ont été appelés à donner une suite au questionnaire en question et ont rechigné à le faire, n'ont pas été cités dans le rapport.

Dans ce contexte, le rapport reste limité en l'absence de réponses émanant des administrations qui sont en contact permanent et quotidien avec les usagers du service public, notamment le Ministère de l'intérieur, le Ministère de la justice et des libertés, l'Administration des douanes et impôts indirects (ADII), la Direction générale des impôts (DGI), la Trésorerie générale du Royaume (TGR), les Universités (...).

\section{II- Contenu}

Dans son contenu, le rapport de la Cour des comptes est édifiant dans les constats et les recommandations qu'il a relevés (1), mais sa teneur présente néanmoins certaines limites non moins importantes (2).

\section{1- Apports}

Le rapport de la Cour des comptes a le mérite d'apporter des éclairages sur la question des services publics en ligne, en analysant notamment la disponibilité et la maturité de ces derniers. À cet effet, la Cour s'est attelée sur des aspects importants relatifs à ces deux problématiques, dont le niveau d'ouverture des données publiques; l'ergonomie des portails institutionnels; la disponibilité et la maturité des principaux services en ligne au niveau national ;leur gouvernance et le suivi par l'administration de leur utilisation et impact.

Ainsi et partant d'une cartographie élaborée par le Ministère de la réforme de l'administration et de la fonction publique, ayant montré une évolution du nombre total des sites web des administrations et établissements publics au Maroc passant de 224 en 2008 à 388 en 2014 soit une augmentation de 73\%, la Cour des comptes a relevé qu'un tel progrès est imputable essentiellement aux services interactionnels ${ }^{1}$ et semi-transactionnels ${ }^{2}$, bien plus que les services informationnels ${ }^{3}$, transactionnel $s^{4}$ et intégrés ${ }^{5}$, tout en constatant que «la mise en

${ }^{1}$ Services permettant d'obtenir de l'information à base d'une requête dynamique. Voir : Cour des comptes, Évaluation des services publics en ligne: Synthèse, op. cit., p. 3.

2 Services permettant d'initier une transaction avec une institution et la compléter manuellement, par poste,... (sans livraison électronique du service). Voir : Cour des comptes, Évaluation des services publics en ligne : Synthèse, op. cit.,p. 3.

3 Services permettant d'obtenir de l'information à base d'une navigation statique non interactive. Voir:Cour des comptes,Évaluation des services publics en ligne : Synthèse, op.cit.,p. 3.

${ }^{4}$ Services permettant d'effectuer une transaction en ligne, de bout en bout, avec une institution (avec livraison électronique du service). Voir : Cour des comptes, Évaluation des services publics en ligne : Synthèse, op.cit., p. 3.

${ }^{5}$ Services permettant d'effectuer une transaction en ligne, de bout en bout, avec plusieurs institutions (avec livraison électr onique du service).Voir : Cour des comptes, Évaluation des services publics en ligne : Synthèse, op. cit.,p. 3. 
ligne de certains services importants reste peu développée par rapport aux ambitions annoncées au programme e-Gov qui couvrait la période 2009-2013, tels les services liés à l'état civil, la création en ligne de l'entreprise et l'immatriculation automobile en ligne ${ }^{1} »$.

En termes de disponibilité et de maturité ${ }^{2}$ des services publics en ligne au Maroc, la Cour des comptes a démontré, en se basant sur un benchmark de l'egouvernement réalisé annuellement par la Commission européenne, que, comparativement aux pays européens, le Maroc « réalise aussi de bons niveaux de maturité sur 8 services, dont les services liés aux impôts (IR, IS et TVA) et droits de douane, alors que 7 autres services dont l'immatriculation d'une nouvelle entreprise et des voitures, les prestations sociales et les documents personnels (passeport, permis de conduire) restent loin de la moyenne européenne dans leurs niveaux de maturité, et sont ainsi, en écart significatif par rapport aux bonnes pratiques en la matière ${ }^{3}$ ». Dans ce cadre aussi, la Cour des comptes a examiné, en puisant dans le benchmark européen, la disponibilité des services publics en ligne représentant six événements de vie (la perte et la recherche d'un emploi ; l'entame d'une procédure courante de plainte; la possession et la conduite d'une voiture ; la poursuite d'études dans un établissement d'enseignement supérieur ; la création d'une entreprise et la réalisation de ses opérations régulières), «considérés par la Commission européenne comme couvrant des domaines parmi les plus courants pour le citoyen et l'entreprise ». Elle a pu mettre en relief le fait que « parmi les 74 services s'insérant dans ces évenements de vie, seuls 33 sont disponibles en ligne, soit un taux de disponibilité global de $45 \%$. Ce taux est de $40 \%$ seulement pour les services de base qui sont en général les étapes essentielles de l'évènement de vie sans lesquelles l'usager serait incapable de compléter sa démarche (19 parmi 48) et de 54\% pour les services complémentaires (14 parmi 26), lesquels vont au-delà des exigences de base d'un évènement de vie, et sont généralement fournis pour faciliter le parcours de l'usager ${ }^{4} »$.

${ }^{1}$ Cour des comptes, Évaluation des services publics en ligne : Synthèse, op. cit., p. 3.

2 Le niveau de maturité reflète la façon dont les entreprises et les citoyens peuvent interagir avec les autorités publiques. Le modèle utilisé pour évaluer la maturité des services en ligne est un modèle à cinq niveaux : informationnel ; téléchargement de formulaires ; traitement des formulaires en ligne ; traitement intégral du cas en ligne ; délivrance automatique du service (sans que l'usager n'ait à réaliser aucune formalité). Définition donnée par la Cour des comptes dans : Évaluation des services publics en ligne: Synthèse,op. cit., p. 4.

${ }^{3}$ Cour des comptes, Évaluation des services publics en ligne: Synthèse, op. cit.,p. 4.

${ }^{4}$ Ibid., p. 5. 


\section{0 المدر 64 ـ مايري}

S'agissant de l'ergonomie des sites web publics, c'est-à-dire leur adaptation aux besoins des utilisateurs, la Cour des comptes a révélé, à partir d'un échantillon de services en ligne composé de 35 services et 9 portails institutionnels, des «faiblesses en matière de disponibilité de fonctionnalités de support dans les services en ligne pour faciliter l'interaction avec l'usager, notamment des forums spécifiques/médias sociaux, des foires aux questions (FAQ), des démonstrations (vidéos en ligne, guides téléchargeables) et des mécanismes de feedback (recueil de l'opinion de l'usager) ${ }^{1}$ ». De plus, la Cour a relevé « le faible taux des services indiquant la durée prévisionnelle de la transaction en ligne (27\%), et la durée maximale de traitement (en back office) de la démarche relative au service $(13 \%)^{2} »$, ainsi que «le manque d'informations sur la dernière mise à jour des pages et la non publication des données publiques en formats facilement réutilisables ${ }^{3}$ ».

Sur le plan de la gouvernance des services en ligne, la Cour des comptes a pu déceler un retard dans l'adoption d'une nouvelle stratégie depuis 2013, date à laquelle le plan Maroc Numeric 2013 est arrivé à son terme, l'insuffisance des prérogatives et les rôles peu clarifiés des organes constitués pour assurer une gouvernance de la stratégie numérique (le Comité national des technologies de l'information et de la société numérique et le Comité interministériel e-Gouvernement), ainsi que le faible suivi de la qualité des services publics en ligne offerts par l'administration.

Concernant le niveau d'ouverture des données publiques (Open Data), la Cour a mis en exergue le faible degré d'ouverture de ces données, lesquelles sont généralement «publiées soit dans des formats inexploitables par la machine, soit de manière discontinue dans le temps ou ne permettant pas leur téléchargement en bloc, voire elles ne sont pas publiées sous licence libre ${ }^{4}$ ». À cela s'ajoute « la régression du Maroc dans les classements internationaux en matière d'Open Data, l'absence de politique d'ouverture des données et le retard accusé dans la réforme du cadre légal, lequel n'englobe aujourd'hui que la loi n09-08 sur la protection des données personnelles et la loi n³1-13 sur le droit d'accès à l'information, et enfin l'absence d'initiatives d'ouverture de données au niveau des collectivités territoriales ${ }^{5} \%$.

${ }^{1}$ Ibid., p. 8.

IIbid.

3lbid.

${ }^{4}$ Ibid., p. 11.

5Ibid., p. 12. 


\section{0 العدد 64 ـ هايرو}

\section{هركز جيل البصث المانمي}

En outre, la Cour des comptes a mis en avant le faible taux de mise à jour par les administrations du contenu du portail "service-public.ma" qui « constitue un point de référence pour accéder, via internet, aux services de l'administration marocaine ${ }^{1} »$; la multiplicité des intervenants en matière de développement des services en ligne et l'inexistence $\mathrm{d}^{\prime}$ « indicateurs nationaux mesurant l'utilisation, l'impact et le degré de satisfaction des usagers de ces services ${ }^{2} »$.

In fine, la Cour des comptes a formulé dans son rapport de nombreuses recommandations visant à faire évoluer le champ des services publics en ligne au Maroc, dont celles consistant à « développer une stratégie numérique détaillée et procéder à sa diffusion en veillant à l'intégration des principaux projets de services en ligne des différents départements ; repenser la gouvernance globale des services publics en ligne et plus particulièrement la relation entre l'Agence de développement du digital et les différents départements, notamment ceux en charge de la fonction publique et de l'intérieur; mettre le citoyen au centre des services publics et focaliser les efforts sur les services en ligne les plus demandés, en adoptant une approche par "événements de vie", retraçant l'ensemble du parcours de l'usager; et faire des évaluations régulières du degré d'utilisation de ces services et de leur impact sur l'usager et sur l'administration ${ }^{3} »$.

\section{2- Limites}

Si le rapport de la Cour des comptes sur l'évaluation des services publics en ligne se distingue par ses nombreux apports, il n'en demeure pas moins qu'il présente des limites dans son contenu qui n'entament pas cependant sa cohérence.

Àce titre, l'éducation des citoyens à l'utilisation et la réutilisation des données n'a pas été mise en avant comme recommandation par le rapport de la Cour des comptes. Or, cette éducation qui a pour objectif de former les citoyens de demain, dès leur bas âge, à «s'emparer des données et qu'ils y soient formés notamment dans un cadre scolaire ${ }^{4}$, passe inéluctablement par «le développement de dispositifs types "infolabs", espaces hybrides où techniciens et citoyens interagissent pour apprendre à utiliser les données; la mobilisation des espaces publics numériques (EPN) pour réaliser cette "éducation aux données", voire aussi l'exploitation des

${ }^{1}$ lbid.

IIbid., p. 13.

3lbid., p. 14.

${ }^{4}$ Samuel Goëtaet Clément Mabi. «L'open data peut-il (encore) servir les citoyens ? », Mouvements, vol. 79, no. 3, 2014, pp. 9091. 
données disponibles en open data dans les programmes scolaires pour illustrer par des cas réels des problèmes rencontrés en classe ${ }^{1} »$.

De même et bien que constituant des instruments de référence en matière de promotion de l'ouverture des données et d'accès aux informations fournies par les services publics, la Convention d'Aarhus sur l'accès à l'information, la participation du public au processus décisionnel et l'accès à la justice en matière d'environnement du 25 juin 1998, ou les principes de Johannesbourg du 1er octobre 1995 sur la sécurité nationale, la liberté d'expression et l'accès à l'information ${ }^{2}$ n'ont pas été abordés dans le rapport. Le même constat peut être relevé s'agissant de certains engagements internationaux touchant indirectement le droit d'accès aux données détenues par les autorités publiques et qui n'ont pas été mentionnés dans ce rapport, à I'instar de la Déclaration universelle des droits de l'homme et le Pacte international relatif aux droits civils et politiques qui reconnaissent dans leur art. 19 le droit de toute personne de « chercher, recevoir et répandre (...) les informations par quelque moyen d'expression que ce soit», ainsi que la Convention des Nations Unies contre la corruption qui oblige, dans son art. 10, les États parties dont le Maroc à prendre les mesures nécessaires pour accroître la transparence de l'administration publique, y compris en ce qui concerne son organisation, son fonctionnement et ses processus décisionnels. Ces mesures incluent d'un côté « l'adoption de procédures ou de règlements permettant aux usagers d'obtenir, s'il y a lieu, des informations sur l'organisation, le fonctionnement et les processus décisionnels de l'administration publique, et de l'autre la simplification, s'il y a lieu, des procédures administratives afin de faciliter l'accès des usagers aux autorités de décision compétentes ».

Par ailleurs, la Cour des comptes qui a privilégié la catégorisation des services publics en ligne en services interactionnels, transactionnels, semi-transactionnels, informationnels et intégrés, a fait abstraction des plateformes publiques en ligne conçues au titre de l'e-participation ${ }^{3}$, notamment le service offert sur le portail http://fikra.egov.ma/, créé pour recueillir les suggestions des usagers pour améliorer l'administration, ou bien d'une part l'initiative mise en œuvre depuis plusieurs années par le Secrétariat général du gouvernement (SGG) qui a mis à la disposition des citoyens le moyen numérique pour exprimer leurs points de vue et soumettre

${ }^{1}$ Ibid.

${ }^{2}$ Disponible en ligne sur <https://www.article19.org/data/files/medialibrary/1803/Johannesburg-Principles.Fra.pdf >.

${ }^{3}$ L'e-participation ou la participation en ligne consiste à favoriser l'engagement des citoyens et à les amener à une gouvernance participative au moyen des technologies de l'information et de la communication (TIC). Voir $<$ https://publicadministration.un.org/fr/eparticipation>. 


\section{0 العدد 64 ـ هايرو}

\section{هركز جيل البصث المانمي}

leurs propositions ${ }^{1}$ au sujet de nombreux projets de lois importants, et de l'autre le portail (www.stopcorruption.ma) qui permet aux citoyens de dénoncer tout acte de corruption.

Concernant les données tirées des classements internationaux, le rapport de la Cour a mis en avant les scores obtenus et les positions occupées par le Maroc au titre de l'indice des Nations Unies EGDI, notamment dans ses deux volets relatifs à l'indice des services en lignes et de l'e-gouvernement, ainsi que de l'indice NRI (Networked ReadinessIndex) établi par le Forum économique mondial. En revanche, aucune mention sur l'indice d'innovation global n'a été incluse dans le rapport. Cet indice qui est élaboré chaque année par I'Organisation mondiale de la propriété intellectuelle (OMPI), mesure la performance des pays en matière d'innovation en prenant en compte 80 indicateurs dont un indicateur sur les services en ligne gouvernementaux (Government's online services) et un autre sur l'e-participation ou la participation en ligne des citoyens, en termes desquels le Maroc a été classé respectivement en $2018^{2} 36^{\text {ème }}$ et $17^{\text {ème3 }}$ sur 126 pays.

\section{Conclusion}

En somme, on peut conclure sur deux considérations principales. Tout d'abord et tel que cela ressort du rapport de la Cour des comptes, le Maroc a réalisé un bond en avant dans l'indice de développement de l'egouvernement (EGDI) et particulièrement dans l'indicateur OSI relatif aux services en ligne où il est passé de la $115^{\text {ème }}$ place/ 182 pays en 2008, à la $78^{\text {ème }}$ place/ 193 pays en $2018^{4}$, mais il convient cependant de « considérer ce progrès avec précaution en tenant compte de l'effectivité de l'usage des plateformes publiques d'eparticipation ${ }^{5}$ »,ainsi que de la nécessité de compléter ces données « par une vision plus affinée des pratiques effectives en la matière et qui pourrait résulter des enquêtes conduites entre autres auprès des responsables $d^{\prime}$ entreprises ${ }^{6} »$ recourant aux services publics en ligne, ainsi qu'auprès des citoyens qui en sont les premiers usagers.

\footnotetext{
${ }^{1}<$ http://www.sgg.gov.ma/arabe/Accueil/Suggestions.aspx $>$.

$2<$ https://www.wipo.int/publications/fr/details.jsp?id=4330\#>.

3<https://www.wipo.int/edocs/pubdocs/en/wipo_pub_gii_2018-profile32.pdf>.

${ }^{4}$ Cour des comptes, Évaluation des services publics en ligne : Synthèse, mai 2019, p. 5.

${ }^{5}$ Institut Royal des études stratégiques, Transformation numérique et maturité des entreprises et administrations publiques, Rapport du 5 septembre 2017, p. 82. Disponible en ligne sur <http://www.ires.ma/wp-content/uploads/2017/11/RAPPORTTRANSFORMATION-NUMERIQUE.pdf>.

${ }^{6}$ lbid.
} 


\section{0}

\section{هركز جيل البمث الهـمي}

En outre, il y a lieu de remarquer que si le développement tous azimuts d'une administration numérique participe de la performance du service public et du rapprochement entre l'administration et ses usagers, il n'en reste pas moins qu'une telle évolution est susceptible aussi de provoquer des distorsions dans la mesure où « la disparition du guichet ou le passage complet aux services en ligne feraient naître un risque de "fracture numérique" entre les individus connectés et les autres ${ }^{1} »$.

\section{Bibliographie}

1. Algan Yann, Bacache-Beauvallet Maya et Perrot Anne. «Administration numérique », Notes du conseil d'analyse économique, vol. 34, no. 7, 2016

2. Agence nationale de réglementation des télécommunications, Usages des TIC dans les ménages et par les individus, Année 2017 : Synthèse des résultats, septembre 2018.

3. Andersen T., «E-Government as an Anti-Corruption Strategy », Information Economics and Policy, $\mathrm{n}^{\circ} 21$, 2009.

4. Cour des Comptes, Évaluation des services publics en ligne, mai 2019.

5. Goëta Samuel et Mabi Clément. «L'open data peut-il (encore) servir les citoyens ? », Mouvements, vol. 79, no. 3, 2014 .

6.Institut Royal des études stratégiques, Transformation numérique et maturité des entreprises et administrations publiques, Rapport du 5 septembre 2017. 